\title{
Effective connectivity analysis of global and local mental imagery by dynamic causal modeling Jian $\mathrm{Li}^{1}$, Danni Sui ${ }^{1}$ and Yi-Yuan Tang*1,2
}

\author{
Address: ${ }^{1}$ Institute of Neuroinformatics and Laboratory for Brain and Mind, Dalian University of Technology, Dalian 116024, China and \\ ${ }^{2}$ Department of Psychology, University of Oregon, Eugene, OR 97403, USA \\ Email: Yi-Yuan Tang* - yiyuan@uoregon.edu \\ * Corresponding author
}

from Seventeenth Annual Computational Neuroscience Meeting: CNS*2008

Portland, OR, USA. 19-24 July 2008

Published: I I July 2008

BMC Neuroscience 2008, 9(Suppl I):P34 doi:10.1 I86/I47|-2202-9-SI-P34

This abstract is available from: http://www.biomedcentral.com/I47I-2202/9/SI/P34

(c) $2008 \mathrm{Li}$ et al; licensee BioMed Central Ltd.

\section{Background}

Despite the large amount of studies on mental imagery, only a few studies have been carried out to investigate the generation of global and local imagery in the brain. To investigate the neural correlates of global-local imagery, event-related potentials (ERPs) were used to measure neuronal responses during generation of global or local mental imagery in 12 healthy subjects. Subjects were shown one target image and then requested to generate the corresponding mental image (global or local). ERPs were recorded for each subject. To further explore the interregional coupling within the global and local imagery tasks, the dynamic causal modeling (DCM) method was recruited. DCM of evoked responses treats the brain as a nonlinear dynamical input-state-output system. In DCM, an experiment is regarded as a designed perturbation of neuronal dynamics that is propagated throughout a network of interconnected anatomical nodes. Effective connectivity is parameterized in terms of coupling among unobserved brain states. The coupling between regions is estimated by perturbing the system using a series of inputs and then measuring the responses. Based on a previous study, we chose the occipito-temporal, parietal and frontal regions as the nodes for effective connectivity analysis $[1,2]$. The position of cortex was adjusted using the dipole localization of SPM5. Effective connectivity analysis was performed using the DCM toolbox in SPM5. The DCM toolbox is distributed with the release of SPM software http://www.fil.ion.ucl.ac.uk/spm.

\section{Results and conclusion}

The occipito-temporal, parietal and frontal regions were actively involved in the generation of global and local imagery in the brain and the right hemisphere played an important role during the process. The task-sensitive connections among the frontal, temporal, supramarginal and cuneus regions were mediated significantly by global and local imagery tasks. In global imagery tasks, the task-sensitive connections were found in both hemispheres whereas the interregional coupling was only detected in left hemisphere for local imagery generation. Our results indicated a distinct neural pathway or effective connectivity existed in the processing of generation of global and local imagery. The two types of mental imagery may derive from different brain mechanism. Our results support the hypothesis that global imagery has a much largerscale processing in the whole brain, whereas the local imagery tends to be left hemisphere specifically.

\section{Acknowledgements}

This work was supported in part by National Natural Science Foundation of China Grant 30670699, Ministry of Education Grant NCET-06-0277 and 021010 .

\section{References}

I. David O, Kiebel SJ, Harrison LM, Mattout J, Kilner JM, Friston KJ: Dynamic causal modeling of evoked responses in EEG and MEG. Neurolmage 2006, 30(4): I255- 1272.

2. Mechelli A, Price C], Friston KJ, Ishai A: Where bottom-up meets top-down: neuronal interactions during perception and imagery. Cerebral Cortex . 2004, June 10, doi: 10.1093 